\title{
In silico prediction and experimental validation of natural antisense transcripts in two cancer-associated regions of human chromosome 6
}

\author{
LAURA MONTI $^{*}$, RAFFAELLA CINQUETTI ${ }^{1 *}$, ALESSANDRO GUFFANTI ${ }^{2}$, FRANCESCO NICASSIO $^{3}$, \\ MATTIA CREMONA ${ }^{4}$, GIOVANNI LAVORGNA ${ }^{5}$, FABRIZIO BIANCHI ${ }^{3}$, FRANCESCA VIGNATI ${ }^{1}$, \\ DAVIDE CITTARO $^{6}$, ROBERTO TARAMELLI ${ }^{1}$ and FRANCESCO ACQUATI ${ }^{1}$ \\ ${ }^{1}$ Department of Biotechnology and Molecular Sciences, University of Insubria, Via JH Dunant 3, I-21100 Varese; \\ ${ }^{2}$ Nanotechnologies Group, Institute of Biomedical Technologies, CNR, Via Fantoli 16/15, I-20138 Milano; \\ ${ }^{3}$ IFOM - FIRC Institute of Molecular Oncology, Via Adamello 16, I-20139 Milano; ${ }^{4}$ Proteomic Unit, \\ Department of Experimental Oncology, National Institute of Cancer, Via Venezian 21, I-20133 Milano; ${ }^{5}$ DIBIT, \\ San Raffaele Hospital, Via Olgettina 58, I-20132 Milano; ${ }^{6} \mathrm{HPC}$ and Bioinformatics Systems @ Informatics Core, \\ FIRC Insitute of Molecular Oncology (IFOM), Via Adamello 16, I-20139 Milano, Italy
}

Received August 28, 2008; Accepted October 27, 2008

DOI: 10.3892/ijo_00000237

\begin{abstract}
Antisense transcription has long been recognized as a mechanism involved in the regulation of gene expression. Therefore, several human diseases associated with abnormal patterns of gene expression might display antisense RNAmediated pathogenetic mechanisms. Such issue could be particularly relevant for cancer pathogenesis, since deregulated gene expression has long been established as a hallmark of cancer cells. Herein, we report on a bioinformatic search for antisense transcription in two cancer-associated regions of human chromosome 6 (6q21 and 6q27). Natural antisense transcripts (NATs) for several genes in both genomic regions were predicted in silico and subsequently validated by strand-specific RT-PCR. Detailed experimental validation by quantitative real-time RT-PCR of five putative cancer related sense-antisense transcript pairs revealed a single candidate tumor suppressor gene (RPS6KA2) whose expression levels display marked cancer-related changes that are likely mediated by its antisense RNA in a breast cancer cell line model.
\end{abstract}

\section{Introduction}

Deletions in the long arm of chromosome 6 (6q) are among the most frequent chromosome aberrations observed in several

Correspondence to: Professor Francesco Acquati, Dipartimento di Biotecnologie e Scienze Molecolari, Via JH Dunant 3, I-21100 Varese, Italy

E-mail: francesco.acquati@uninsubria.it

${ }^{*}$ Contributed equally

Key words: chromosome 6, antisense RNA, tumor suppressor genes, transcriptional regulation human malignancies, such as non-Hodgkin's lymphomas, melanoma and carcinomas of the mammary gland, ovary, uterus, stomach, kidney and salivary gland (1-3). The involvement of this chromosome in the pathogenesis of the abovementioned cancer types has been demonstrated by LOH studies and chromosome-transfer assays, which led to the identification of several regions of minimal deletion spanning quite large segments of genomic DNA (4-6). More specifically, independent studies have been published in the last decade reporting a frequent involvement of the $6 \mathrm{q} 21$ and 6 q27 genomic regions in cancer-related chromosomal rearrangements $(1,2)$, raising the hypothesis that one or probably more tumor suppressor genes (TSGs) reside in these regions. Several genes mapping in 6q21 and 6q27 have been recently evaluated as putative TSGs. Among these, the BLIMP1/PRDM1, RPS6KA2 and RNASET2 genes have been implicated in the pathogenesis of single tumor types (7-9). However, TSGs involved in the wide range of human cancers associated with $6 \mathrm{q} 21$ and $6 \mathrm{q} 27$ rearrangements have escaped detection to date despite intensive investigations (1012), the only exception being perhaps the HACE1 gene (13). Interestingly, several genes from 6q27 that have been recently evaluated as candidate TSGs did not show inactivating coding mutations in cancer cells; rather, tumor-associated downregulation of the corresponding transcripts was observed for these genes (8-10), raising the possibility that they could be involved in cancer pathogenesis acting as epigenetically silenced class II TSGs. Quite unexpectedly, however, the promoter methylation pattern for these genes was shown to be unchanged in cancer cells, suggesting that other mechanisms besides epigenetic modification should be envisioned to explain the decreased expression levels observed for these putative class II TSGs in cancer cells.

Natural antisense transcripts (NATs) are endogenous transcripts containing sequences complementary to other endogenous mRNAs, which are usually associated with a known 
function and are therefore defined as sense RNAs $(14,15)$. Following the completion of the human genome sequence, several in silico studies, coupled with microarray analysis, have demonstrated that the occurrence of NATs is quite widespread in the human genome $(16,17)$, with the most recent surveys suggesting that up to $70 \%$ of the human transcriptional units have natural antisense partners (18-20). Antisense transcription has been involved in the regulation of mammalian gene expression by means of several mechanisms, including transcriptional interference, RNA-masking, RNA editing, double stranded RNA (dsRNA)-dependent mechanisms and antisense induced promoter methylation (15). Significantly, aberrant gene expression has been identified as an important molecular mechanism involved in cancer progression $(21,22)$. Moreover, the widespread DNA hypomethylation usually observed in the cancer cell genome has been recently proposed to trigger a global transcriptional de-repression wave, thus generating a strong transcriptional 'noise' (23). By this mechanism, antisense transcripts with aberrant regulation would be produced, some of which could affect the function of overlapping TSGs and oncogenes, ultimately paving the way for cancer progression.

Indeed, a recent report by $\mathrm{Yu}$ et al showed that an impressive proportion of the well known TSGs likely possess nearby antisense RNAs (24). Moreover, antisense-mediated downregulation for one of these TSGs (p15) was experimentally confirmed in leukaemia cells. Thus, the hypothesis of NATs acting as effectors of aberrant gene expression in cancer cells is of particular interest and justifies a targeted search for antisense transcription within genomic regions which have been shown experimentally to harbour cancerrelated genes. However, although antisense-mediated regulation of cancer-related genes has been analyzed in a handful of reports focusing on a single locus (25-27), very few studies have been devoted to a systematic search for antisense genes putatively involved in cancer pathogenesis.

Here, we report both in silico and experimental evidence for the occurrence of antisense transcription in the $6 \mathrm{q} 21$ and $6 q 27$ chromosomal regions. Interestingly, some of the NATs identified in this work were shown to target 'sense' genes whose function make them plausible candidates for cancer pathogenesis. Indeed, detailed characterization of one such sense/antisense pair provided compelling evidence for antisense-mediated transcriptional deregulation of the RPS6KA2 tumor suppressor gene, using mammary adenocarcinoma as an experimental model.

\section{Materials and methods}

RNA, cell lines and culture conditions. Total RNA from normal human fetal brain and adult breast was purchased from Stratagene and Invitrogen, respectively. Total RNA from cultured cell lines was prepared with the TRI-reagent (Sigma) following the manufacturer's instructions. The breast cancer cell lines MDA-MB 213, MDA-MB 453, MCF-7, HBL100, BT20 and T47D were cultured in Iscove's medium supplemented with $10 \%$ fetal bovine serum.

Bioinformatic analysis. The in silico search for antisense transcripts was performed with the 'AntiHunter' online anti- sense detection tool (http://bioinfo.crs4.it/AH2.0/) (28). Briefly, a catalogue of all genes available in the public human genome annotation databases was first assembled for the two chromosomal regions of interest (6q21 and 6q27). The elements retrieved included transcript identifiers, gene coordinates, strand of the corresponding transcripts, description of the gene product, informations on mouse orthologs and expression pattern. The genomic sequences corresponding to the annotated genes were retrieved with an extension of 1500 base pairs at both ends of the annotated gene transcript. We then used a series of perl scripts to produce a relational 'Antisense' database, collecting all the gene-related information. The AntiHunter search output included a variety of information related to the putative antisense ESTs, including accession number, match significativity, strand identity, presence of splice site signals and expression pattern. To further refine the results, we used the CAP Assembler (http://bio.ifom-ieocampus.it/cap) to produce contigs of antisense EST sequences that were remapped to the genome assembly consensus. We then installed a local copy of the GBrowse Generic Genome Browser (http://www.gmod.org/ggb/) and linked it with the Antisense database, to provide an intuitive way for the location of the antisense EST sequences in the genome context. A database with the results of the bioinformatics procedure is freely available at the web link http://bio.ifom-ieo-campus.it/ antisense.

Strand-specific and quantitative real-time RT-PCR. Total RNA was extracted using TRI-Reagent (Sigma) following the manufacturer's protocol. For all RT-PCR reactions performed, RQ-DNAse (Promega) treatment of RNA samples was carried out and a PCR assay on DNAse-treated RNA was performed to rule out DNA contamination. One microgram of total RNA was used for the strand-specific RT-PCR assays. The same primer pair was used to detect both sense and antisense transcription from a single locus by selecting it from the overlapping region between the antisense ESTs and the genomic DNA or the mRNA of the corresponding sense gene (with reference to the NCBI release 34). When an antisense EST contig was present, the best EST vs. genome match was selected for primer selection. Primer sequences for all sense-antisense gene pairs that have been experimentally validated are available on request.

Strand-specific RT-PCR reactions were carried out using the Sigma DuraScript ${ }^{\mathrm{TM}}$ RT-PCR HSRT-100 kit in a final reaction volume of $25 \mu \mathrm{l}$. Four reactions were set up for every gene to be tested: i) retrotranscription of the antisense transcript: the forward primer only was included during the retrotranscription step; ii) retrotranscription of the sense transcript: the reverse primer only was included during the retrotranscription reaction; iii) positive control: both primers have been used $(0.5 \mu \mathrm{l}$ of a $50 \mu \mathrm{M}$ solution $)$ but samples have been introduced in the thermocycler only during the PCR phase. Genomic DNA was used as a template instead of RNA; iv) negative control: both primers have been added to the reaction mix ( $1 \mu 1$ of a $10 \mu \mathrm{M}$ solution) but no RNA template was included to the reaction mix.

In all four reactions, both sense and antisense primers were added to the reaction mix before the PCR reaction ( 0.5 of a $50 \mu \mathrm{M}$ solution). PCR conditions were as follows: 
the retrotranscription step was performed at $50^{\circ} \mathrm{C}$ for $50 \mathrm{~min}$. Thereafter, a single 5 -min step at $94^{\circ} \mathrm{C}$ was performed, followed by 35 cycles of amplification with $30 \mathrm{sec}$ at $94^{\circ} \mathrm{C}$ (denaturation), $30 \mathrm{sec}$ at a variable annealing temperature, depending on the single primer pair (annealing) and $1 \mathrm{~min}$ at $72^{\circ} \mathrm{C}$ (extension), and a single final extension step of $5 \mathrm{~min}$ at $72^{\circ} \mathrm{C}$. PCR products were analyzed by agarose gel electrophoresis with standard buffers and conditions.

For quantitative real-time RT-PCR assays, the Primer Express Software version 2.0 program (Applied Biosystems) was used to design, for each gene to be tested, forward and reverse primers having the same annealing temperature and GC content. Total RNA $(1 \mu \mathrm{g})$ together with each specific primer at a final $0.5 \mu \mathrm{M}$ concentration, was heated at $65^{\circ} \mathrm{C}$ for $5 \mathrm{~min}$, chilled on ice and incubated at room temperature for $10 \mathrm{~min}$; the RNA was then retrotranscribed (in triplicate) using the High Capacity cDNA synthesis kit (Applied Biosystems) for $2 \mathrm{~h}$ at $37^{\circ} \mathrm{C}$. Real-time RT-PCR was performed on ABI PRISM 7000 with Power SYBR green PCR Master Mix (Applied Biosystems) following the manufacturer's instructions. The sequence of all primer pairs used are available on request. Following a DNA polymerase activation step at $95^{\circ} \mathrm{C}$ for $10 \mathrm{~min}$, samples were denaturated at $95^{\circ} \mathrm{C}$ for $12 \mathrm{sec}$ and annealed/extended at $60^{\circ} \mathrm{C}$ for $1 \mathrm{~min}$, for $35 / 45$ cycles. Fluorescent signals generated during PCR amplification were monitored and analyzed with ABI PRISM 7000 SDS software (Applied Biosystems). Comparison of the amount of each gene transcript among different samples was made using $B$-actin as reference. The amount of target RNA, normalized to the endogenous reference gene, was calculated by means of the difference-in-threshold-cycle parameter $\left(\Delta \mathrm{C}_{\mathrm{t}}\right)$.

RNAi and transcript overexpression for RPS6KA2 sense/ antisense gene pair. BT20 and T47D cells were transfected for RNA interference either once with $100 \mathrm{nM}$ or twice (at 24- or 48-h time interval) with $50 \mathrm{nM}$ RPS6KA2-specific On-Target plus Duplex J-004663-06 or J-004663-07 scrambled control oligo (Dharmacon) using Oligofectamine (Invitrogen Corp.) according to the manufacturer's instructions. The cells were then collected at $48 \mathrm{~h}$ post-transfection for real-time RT-PCR analysis. A 674-bp DNA fragment corresponding to an experimentally confirmed overlapping region between RPS6KA2 and its antisense transcript (genomic coordinates: 166.919.038-166.919.711 - UCSC assembly March 2006) was cloned by RT-PCR in pcDNA3 (Invitrogen) with an orientation resulting in transcription of the antisense RNA. BT20 cells were seeded two days before performing cotransfection with pcDNA3-antisense or empty vector together with pCMV-GFP (5:1) as a reporter construct. Two days after transfection with Fugene (Roche), cells were collected and GFP-sorted in order to improve the detection of transfected genes. Real-time RT-PCR for RPS6KA2 sense/antisense RNA was performed as described above.

Microarray data analysis. Expression data for 59 lymph nodenegative (N0) estrogen receptor-positive (ER+) breast cancer patients, previously generated with Affymetrix HG-U133 chip A+B $(29,30)$, were extracted for the RPS6KA2 sense (probeset: 204906_AT) and antisense (probeset: 225210_S_AT) tran- scripts. Raw data was median centred and $\log 2$ converted, in order to determine the reciprocal correlation between the two genes (positive: up/up or down/down; negative: up/down or down/up). P-value was calculated with Fisher's exact test with the JMP 7.0.1 software (SAS).

\section{Results}

In silico detection of NATs from 6q21 and 6q27. As a first step towards the identification of sense-antisense transcript pairs from $6 \mathrm{q} 21$ and $6 \mathrm{q} 27$, we performed an extensive in silico search for putative NATs from both chromosomal regions. To this aim, a collection of well-annotated genes was retrieved from public human genome annotation resources and assembled into a dedicated database. A total of 101 genes (61 genes from 6 q21 and 40 genes from 6 q27) were reported on both genomic regions; the genomic DNA sequence corresponding to these annotated genes was therefore retrieved, transferred into our database and used as the input data for the identification of antisense transcripts by means of the AntiHunter web tool.

This analysis produced an initial output of 24 genes (20 from 6 q21 and 4 from $6 q 27$ ) for which antisense EST sequences were reliably detected in silico (Table I). A total of 182 antisense ESTs were found for the 4 genes in $6 \mathrm{q} 27$, whereas 453 antisense ESTs were detected for the 20 genes in 6q21. All 24 genes had corresponding RefSeq sequences besides AK094715 and c6orf208, which represent in silico predicted genes. Analysis of all antisense ESTs with CAP Assembler showed that it was often possible to assemble a single contig represented by several EST sequences internal to a major longer antisense EST, further suggesting that bona fide antisense RNAs were detected.

The re-alignment of all antisense ESTs on the human genome sequence allowed us to deduce the pattern of overlap between the putative sense and antisense transcript pairs at each locus. Among the 24 sense genes for which AntiHunter predicted the occurrence of antisense transcription, 14 (58\%) turned out to share at least one exon with their putative antisense partner, thus representing sense-antisense (SA) clusters according to the definition of Chen et al (18). For the remaining 10 genes (42\%) the inferred sense-antisense pattern of overlap did not include any exon, therefore these transcript pairs were classified as non-exon overlapping bidirectional clusters (NOBs) (Table I).

The alignment of the in silico predicted antisense ESTs on the human genome showed that the majority of these EST sequences were indeed derived from human chromosome 6 , with an identity percentage ranging from 97 to $99 \%$. This was taken as an indication that most antisense ESTs predicted by the AntiHunter analysis likely represent cis-NATs. Many of the significative alignments to non- 6 chromosomes were due to repetitive elements, therefore the corresponding ESTs were not considered for further analysis. However, for a few genes in the two chromosomal regions under investigations it was not possible to determine unambiguously the locus from which the predicted NATs were transcribed, since the latter produced alignments with identical scores in two or more genomic regions. Thus, some of these antisense transcripts could actually represent trans-NATs (Table I). 
Table I. Summary of 24 annotated genes from $6 \mathrm{q} 21$ and $6 \mathrm{q} 27$ for which antisense transcription was predicted in silico by AntiHunter.

\begin{tabular}{|c|c|c|c|c|c|c|c|}
\hline $\begin{array}{l}\text { Gene } \\
\text { symbol }\end{array}$ & RefSeq & $\begin{array}{l}\text { Human genomic } \\
\text { sequence } \\
\text { coordinates }^{\mathrm{b}}\end{array}$ & $\begin{array}{l}\text { Type of sense- } \\
\text { antisense } \\
\text { gene pair }\end{array}$ & $\begin{array}{l}\text { Region of the sense gene } \\
\text { overlapping with } \\
\text { antisense RNA }\end{array}$ & $\begin{array}{l}\text { Antisense } \\
\text { RNA in } \\
\text { the mouse }\end{array}$ & $\begin{array}{l}\text { Evidence for } \\
\text { coding anti- } \\
\text { sense RNA }\end{array}$ & $\begin{array}{l}\text { Evidence } \\
\text { for trans- } \\
\text { NATs }\end{array}$ \\
\hline
\end{tabular}

\section{6q21}

AK094715 -

ARMC2 NM_032131.4

ATG5 NM_004849.2

CD164 NM_006016.3

CDC2L6 NM_015076.3

FYN NM_002037.3

109664510-109698413

109276318-109401877

106739045-106880388

109794414-109810340

111037874-111243530

112089178-112301320

$\begin{array}{ll}\text { NOB } & \text { 2nd intron } \\ \text { NOB } & \text { 8th intron } \\ \text { AS } & \text { 6th intron and } \text { 3' UTR }^{\prime} \\ \text { AS } & \text { 3' UTR } \\ \text { NOB } & \text { 2nd and 4th intron } \\ \text { AS } & \text { 4th and 12th intron, }\end{array}$

MICAL

NM_022765.2

OSTM1 NM_014028.3

PDSS2 NM_020381.3

POPDC3 NM_022361.3

PRDM1 NM_001198.3

PREP NM_002726.3

QRSL1 NM_018292.3

REV3L NM_002912.3

RTN4IP1 NM_032730.4

SEC63 NM_007214.4

SMPD2 NM_003080.2

SNX3 NM_003795.3

TRAF3IP2 NM_147686.1

TUBE1 NM_016262.3

109871959-109883883

108469306-108502634

107580454-107887472

105712470-105734563

106640888-106664507

105832199-105957662

107184146-107222103

111726927-111911107

107125596-107184066

108295653-108386175

109868624-109871815

108639410-108689156

111986836-112034014

112498673-112515349

AS

11th and 12th exon

AS Exon 23 to 3 ' UTR

AS 3' UTR

NOB 4th intron

NOB 1st intron

NOB 3rd intron

AS 13th exon and 14th intron

NOB 9th intron

AS $\quad \underline{1 s t}, 4$ th, 12th and 27th intron

AS

AS

AS

AS

AS

AS

3' UTR

1 st and 4th intron; $\underline{3^{\prime} \text { UTR }}$

5' UTR

3' UTR

1 st, 2th to 8th intron

3' UTR

$6 \mathrm{q} 27$

$\begin{array}{lllll}\text { C6orf208 } & - & 169932342-169944894 & \text { NOB } & \text { 1st intron } \\ \text { PHF10 }^{a} & \text { NM_133325.2 } & 169845926-169866031 & \text { AS } & \begin{array}{l}\text { 9th intron to 10th exon; } \\ \text { 11th intron to 12th exon }\end{array} \\ \text { RPS6KA2 } & \text { NM_001006932.1 } & 166742844-167195761 & \text { NOB } & \underline{1 \text { st }, 6 \text { th, 7th intron }} \\ \text { SFT2D1 } & \text { NM_145169.1 } & 166653506-166675981 & \text { NOB } & \text { 1st intron }\end{array}$

The genes marked in bold were selected for further experimental validation on the basis of bioinformatic evidence for antisense transcription also in the mouse genome. Each sense-antisense gene pair was classified either as antisense-sense exon overlapping (AS) or non-exon overlapping bidirectional (NOB) gene pair, according to the inferred pattern of overlap between the sense and antisense transcripts. The region in the sense gene which was chosen for PCR detection of antisense transcription is underlined for those gene pairs where several regions of overlap between sense and antisense transcripts were found. Evidence for the putative occurrence of trans-NATs was obtained by genome-wide BLAT analysis with the nucleotide sequence of each putative antisense EST. ${ }^{a}$ The antisense gene for PHF10 is itself a RefSeq gene (c6Orf120 - see text). ${ }^{b} U C S C$ assembly Mar 2006.

The list of genes from 6q21 and 6q27 for which we predicted antisense transcription in silico was compared with the recent on-line catalog of sense-antisense pairs from the Laboratory of Computational Biology at LICR São Paulo, Brasil (http://www.compbio.ludwig.org.br/sense-antisense/) (31). We found evidence for antisense transcription in the LICR database for 14 out of 24 genes from our list, providing a further bioinfomatic support for our results. Moreover, we identified from the UCSC genome browser six Acembly antisense transcripts that reinforced the evidence initially derived from single antisense EST sequences.
Two of the RefSeq genes under investigation (PHF10 and c6Orf120) were found to overlap at their 3' UTRs, thus representing a couple of sense-antisense pair between two protein coding genes (although the gene product from the latter has not yet been experimentally confirmed). Moreover, using the source databases (UCSC, Aceview and LICR) together with the ORF Finder tool (http://www.ncbi.nlm.nih. gov/gorf/gorf.html) to analyze the coding potential of each putative NAT, we found a potential ORF in the antisense transcript of seven sense genes (ARMC2, FYN, PREP, QRSL1, SMPD2, TUBE1, RPS6KA2). For all other sense genes, the 
corresponding in silico predicted antisense transcripts likely represent bona fide non-coding antisense genes.

Thus, the final result of our in silico analysis was an annotated list of 24 genes for which evidence for the occurrence of antisense transcription was found (Table I). In order to define a list of biologically relevant candidate NATs among those detected by AntiHunter, the occurrence of antisense transcription for both the human and murine loci was investigated. This criterion was chosen since evolutionarily conservation of sense-antisense transcript pairs has recently been shown to represent a reliable hallmark of functional relevance for antisense-mediated gene regulation $(33,34)$. In $6 q 27,2$ out of the 4 sense genes for which antisense ESTs were found gave evidence for a corresponding antisense transcript also in the mouse genome. Similarly, in the 6q21 region, 14 out of 20 sense genes showed the presence of an antisense transcript in the mouse (Table I). These 16 evolutionarily conserved and putative antisense-regulated genes were therefore selected for primary biological validation of antisense transcription by means of strand-specific RT-PCR.

Experimental validation of candidate NATs. For each selected sense-antisense gene pair, a couple of primers was designed as described in Materials and methods to detect transcription from both strands on each locus.

Since a large fraction of the human genome is known to be expressed in fetal brain (35), RNA from this tissue was chosen as the template for RT-PCR assays. Moreover, total RNA was used rather than a poly- $\mathrm{A}^{+}$fraction to perform this analysis, since most NATs have been recently reported to represent non-polyadenylated transcripts (36).

As a control, we included in our strand-specific RT-PCR assays two genes from 6q21 (FOXO3A and CDK11) and two from 6q27 (RNASET2 and FOP) for which Antihunter did not detect antisense ESTs. The results of the validation assay is shown in Fig. 1: eleven antisense transcripts on a total of 16 genes tested $(69 \%)$ were detected using just a single tissue as a source of RNA for RT-PCR reactions. As expected, the four control genes for which we could not find in silico evidence of antisense transcription produced an expected amplification band with the sense strand-specific primer only (two of these four genes, FOXO3A and RNASET2, are included in Fig. 1).

Strikingly, for most gene pairs analyzed we observed expression of both sense and antisense transcripts in human fetal brain RNA. Such co-expression pattern was also observed for 5 sense-antisense NOB gene pairs (POPDC3, PRDM1, QRSL1, PDSS2 and RPS6KA2), whose overlapping sequences did not involve known exons from the sense gene. Therefore, since the same primer pair was used to detect transcription from both DNA strands, the sense transcripts detected by RT-PCR for these NOB pairs likely represented either a novel, previously unrecognized exon from the sense gene or its pre-mRNA. Indeed, the latter event was not completely unexpected since, as mentioned before, total RNA rather than the poly- $\mathrm{A}^{+}$fraction was used as a template for our RT-PCR assays. Accordingly, when the RT-PCR assays were repeated using exon-specific primers to detect the sense transcript, expression of the mature sense transcript for all six NOB pairs was confirmed (data not shown).

\begin{tabular}{|c|c|c|c|}
\hline POPDC3 & $1--1$ & SNX3 & $=--$ \\
\hline PRDM1 & $---\bullet$ & CD164 & -0 \\
\hline ATG5 & $-\infty$ & SMPD2 & 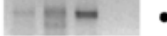 \\
\hline QRSL1 & $\operatorname{sen} \bullet$ & TUBE1 & 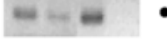 \\
\hline PDSS2 & $=-m=$ & REV3L & -- \\
\hline SEC63 & -- & TRAF3IP2 & $-=$ \\
\hline OSTM1 & $-=$ & FYN & $=0$ \\
\hline RPS6KA2 & 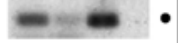 & PHF10 & $-\quad-$ \\
\hline FOXO3A & $=-$ & RNASET2 & -- \\
\hline
\end{tabular}

Figure 1. RT-PCR validation for in silico predicted NATs. AS, antisense transcript; $\mathrm{S}$, sense transcript; +, positive control (total human genomic DNA); -, negative control (no RNA template). A black dot highlights sense genes for which an antisense transcript was experimentally detected. FOXO3A and RNASET2 are control genes for which no antisense transcripts were predicted by AntiHunter.

An issue that has not been given much attention in most studies of antisense transcription is the possibility that RNA self-priming events during the retrotranscription step could result in the false appearance of putative antisense transcripts in an RNA sample of interest. These spurious events can be easily revealed by omitting the strand-specific primer during the retrotranscription step of an RT-PCR reaction. Therefore, a further strand-specific RT-PCR assay with a control reaction (in which the antisense-specific primer was omitted in the retrotranscription step) was carried out for all the sense genes for which antisense transcription was experimentally confirmed. Only 2 out of the 11 selected genes (PDSS2 and SNX3) gave clear evidence of self-priming events, casting some doubt over the real occurrence of their corresponding antisense transcripts (data not shown).

Our strand-specific RT-PCR assays were carried out with fetal brain RNA in order to experimentally detect a number of NATs as large as possible. However, since fetal brain represents a complex mixture of several different cell types, we could not be certain that the observed expression of both sense and antisense RNAs in this tissue truly represented co-expression of both transcripts within a single cell type. This is an important issue, since regulation of gene expression by antisense-mediated mechanisms can only take place when both transcripts of a sense-antisense pair are present in the same cell. Furthermore, fetal brain is a normal tissue, while our study was targeted to the identification of sense-antisense gene pairs with some relevance to cancer pathogenesis. To address both issues, we decided to evaluate the expression pattern of 5 sense-antisense transcript pairs in a panel of six mammary cancer cell lines (MDA-MB231, MDA-MB 453, MCF7, HBL100, BT20 and T47D) by strand-specific RT-PCR. The 5 gene pairs were selected from our list of in silico predicted, experimentally confirmed sense-antisense transcripts on the basis of the established or putative candidacy 
Table II. Genes analyzed by strand-specific RT-PCR and quantitative real-time PCR.

\begin{tabular}{lcrl}
\hline Gene & Location & OMIM entry & \multicolumn{1}{c}{ Gene product's function } \\
\hline FYN & $6 \mathrm{q} 21$ & $* 137025$ & $\begin{array}{l}\text { Membrane-associated tyrosine kinase that has been implicated in the control of cell growth } \\
\text { TUBE1 }\end{array}$ \\
$6 \mathrm{q} 21$ & $* 607345$ & $\begin{array}{l}\text { Tubulin epsilon. The gene product localize within the centrosome of dividing osteogenic sarcoma cells } \\
\text { in a cell cycle-dependent manner }\end{array}$ \\
TRAF3IP2 & $6 \mathrm{q} 21$ & $* 607043$ & $\begin{array}{l}\text { The gene product interacts with TRAF proteins (tumor necrosis factor receptor-associated factors) } \\
\text { and either I-kappa B kinase or MAP kinase to activate either NF-kappaB or Jun kinase }\end{array}$ \\
RPS6KA2 & $6 \mathrm{q} 27$ & $* 601685$ & $\begin{array}{l}\text { Serine/threonine kinases that phosphorylates various substrates, including members of the mitogen- } \\
\text { activated kinase (MAPK) signalling pathway. Implicated in controlling cell growth and differentiation }\end{array}$ \\
PHF10 & $6 \mathrm{q} 27$ & - & $\begin{array}{l}\text { Predicted ORF encoding a protein with two zinc finger domains } \\
\text { Expression data for these genes were obtained in normal breast and a panel of breast cancer cell lines, on the basis of the predicted or experimentally } \\
\text { confirmed involvement for these genes in cellular or biochemical processes related to cancer. }\end{array}$ \\
\hline
\end{tabular}

\section{$\begin{array}{lllll}1 & 2 & 3 & 4 & 5\end{array}$}

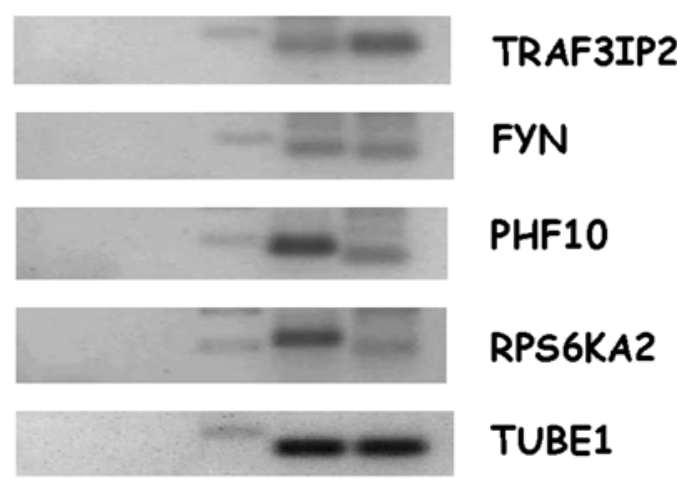

Figure 2. Co-expression of sense and antisense transcripts for five putative cancer-related sense genes within a single cell type (HBL-100 breast cancer cell line) assessed by strand-specific RT-PCR. Lane 1, negative control for antisense transcript; lane 2, negative control for sense transcript; lane 3, molecular weight marker; lane 4, antisense transcript; lane 5, sense transcript The different molecular weight observed for the PHF10 and RPS6KA2 transcripts is due to the fact that different primer pairs were used to detect the sense and the antisense transcript for these genes.

as a cancer-related gene for the sense transcript (Table II). Significantly, for all 5 genes the co-expression of both sense and antisense transcripts previously observed in human fetal brain was confirmed in most cell lines analyzed, thus demonstrating that these five loci do really express in a single cell type both sense and antisense RNAs, which therefore have the potential to interact functionally. Fig. 2 shows the expression data for a single cell line (HBL-100) in which co-expression of both sense and antisense transcripts for all five gene pairs was observed.

Quantitation of sense and antisense RNA levels in a panel of cancer cell lines. The co-expression in different mammary cancer cell lines of both sense and antisense transcripts for the above-mentioned cancer-related genes prompted us to evaluate the biological relevance of this observation.
To this aim, quantitative real-time RT-PCR assays were performed on the same cancer cell line panel described above and on a normal breast tissue sample, in order to detect putative changes in the relative expression levels of sense and/or antisense transcripts. The results of this analysis are shown in Fig. 3. For the five genes tested, different patterns of changes in the expression levels in normal versus cancer cells were observed for either the sense or the antisense transcript (or both). For example, expression of both sense and antisense RNA from the TRAF32IP gene turned out to be significantly increased in all cancer cell lines, when compared to the non-neoplastic counterpart. Since both sense and antisense RNA levels were increased proportionally, the observed changes in the RNA levels from both DNA strands at this locus did not translate in a significant change in the sense/antisense transcript ratio in the cancer cell lines panel. A similar trend was observed for the FYN gene, where a significant change in the sense/antisense transcript ratio was observed in two cell lines only.

As far as the TUBE1 gene is concerned, although significant changes in the levels of either sense or antisense transcripts were found in some tumor cell lines, a clear trend did not emerge when the ratio between sense and antisense expression levels was evaluated.

However, the expression pattern observed for the remaining two genes included in this analysis (PHF10 and RPS6KA2) proved much more interesting. Although we noted a trend for a cancer-specific upregulation of the antisense RNA for PHF10, the sense transcript (e.g., the PHF10 transcript itself) showed a much more dramatic increase in all cancer cell lines examined. Therefore, an increased sense/antisense ratio was observed in 5 out of 6 cell lines for this gene. Although this observation does not necessarily imply a reciprocal regulation between the sense and antisense transcript, the marked upregulation of PHF10 sense RNA in all tumor cell lines examined points to a putative oncogenic role for this gene.

Even more interesting were the results obtained with the RPS6KA2 transcript pairs. In this case, the observed downregulation of the sense transcript in 6 out of 7 cancer cell 


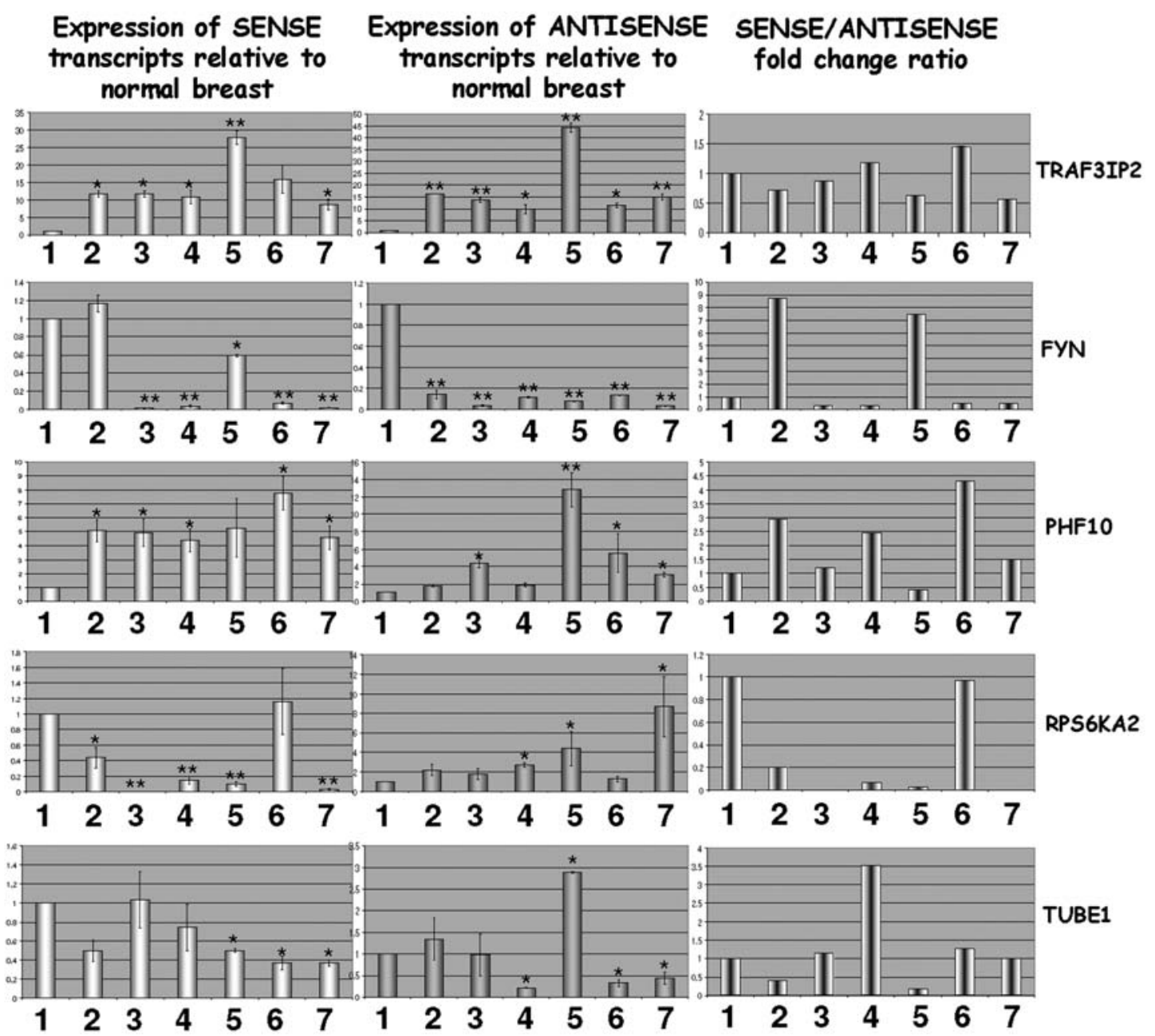

Figure 3. Quantitative real-time RT-PCR for both sense and antisense transcripts from five putative cancer-related genes. The PCR assay was performed as described in Materials and methods. For each gene, the expression of either the sense or the antisense transcript (left and middle panels) in several breast cancer cell lines was compared to that observed in a normal breast control sample following normalization with the $\beta$-actin reference gene. The sense/antisense fold change ratio for cancer cell lines vs. normal breast tissue was then calculated (right panel) by using as a reference the ratio between the sense and antisense transcripts in the normal breast control sample, which was arbitrarily given a value of one. Bars indicate means with SE; ${ }^{*} \mathrm{p}<0.05 ;{ }^{* *} \mathrm{p}<0.01$. Statistical analysis was carried out with One-way ANOVA. Lane 1, normal breast; lane 2, MDA-MB231; lane 3, MDA-MB 453; lane 4, MCF7; lane 5, HBL100; lane 6, BT20; lane 7, T47D.

lines with respect to the normal tissue sample was mirrored by a parallel increase of the corresponding antisense RNA in the same cell lines (for example, normal breast tissue and the BT20 cell line displayed both the higher expression level of sense RNA and the lowest expression level of the antisense RNA within the whole samples panel, whereas the opposite trend was observed for the T47D cell line).

When the sense/antisense expression ratio was examined for this locus, all cancer cell lines showed a significant decrease of this value when compared to the normal breast tissue, with the exception of the BT20 cell line. These results strongly suggest the occurrence of sense-antisense reciprocal gene regulation for this locus.

The antisense transcript for the RPS6KA2 gene could not be unambiguously assigned in the genome, since it produced two alignments with identical scores on chromosomal bands $6 \mathrm{q} 27$ (where the RPS6KA2 locus reside) and 15q25.2. Indeed, whereas in the latter chromosomal band the RPS6KA2 antisense transcript was mapped to a 1200-bp region representing the full-length transcript of the FAM102A1 gene, a processed FAM103A1 pseudogene with antisense orientation to the RPS6KA2 gene was also found in $6 \mathrm{q} 27$. Therefore, the experimentally detected RPS6KA2 antisense RNA could derive from either the spliced transcript of the FAM103A1 gene on chromosome 15 (thus representing a trans-NAT) or, alternatively, from the unspliced RNA of the FAM103A1 processed pseudogene on chromosome 6 (thus representing a cis-NAT) or perhaps from both loci.

To further investigate the functional role of RPS6KA2's antisense RNA in the regulation of the sense transcript levels, we decided to experimentally manipulate the intracellular levels of both sense/antisense transcripts in two of the abovementioned cell lines (BT20 and T47D).

As shown in Fig. 4A, knocking down the RPS6KA2 sense transcript levels by means of RNA interference in the 'highexpressing' cell line BT20 triggered a significant increase in the levels of the corresponding antisense transcript, as assessed by quantitative real-time RT-PCR. Unfortunately, the reciprocal experiment (i.e. knocking down the RPS6KA2 antisense gene transcript in the 'high-expressing' T47D cell 
A

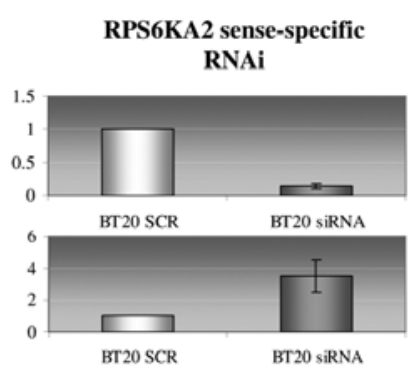

B

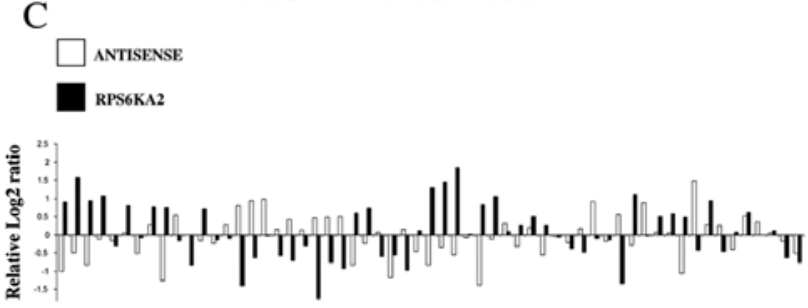

Figure 4. Quantitative real-time RT-PCR for both RPS6KA2 sense and antisense transcripts in the BT20 mammalian cancer cell line following either RNAi against the sense transcript (A) or antisense transcript overexpression (B). The PCR assay was performed as described in Materials and methods. For each gene, the expression of either the sense or the antisense transcript was normalized with the $B$-actin reference gene. Bars indicate means with SE. C, Expression data for RPS6KA2 sense (probeset: 204906_AT) and antisense (probeset: 225210_S_AT) transcript on a panel of 59 breast cancer patients. Raw data were median centered and $\log 2$ converted. P-value (0.0092) was calculated with Fisher's exact test (JMP 7.0.1)

line) was not informative. For unknown reasons we were not able to achieve a significant silencing of the antisense transcript by RNAi in this cell line, despite several attempts with a wide range of experimental conditions (data not shown).

We thus turned again to the BT20 cell line, and decided to see whether overexpression of the RPS6KA2 antisense RNA (whose expression level is very low in this cell line) was associated with a decrease in the intracellular levels of sense transcript. Indeed, a marked downregulation of the RPS6KA2 sense gene expression was observed by real-time RT-PCR in cells transfected with an antisense-expressing vector (Fig. 4B), suggesting antisense-mediated silencing as a molecular mechanism for the regulation of the intracellular levels of the RPS6KA2 transcript.

On the basis of these data, we hypothesized that reciprocal regulation of the sense/antisense transcript pair for the RPS6KA2 locus may also occur in primary human cancer specimens. Therefore, based on our previous results with human breast cancer cell lines, we extracted the data for RPS6KA2 sense (probeset: 204906_AT) and antisense transcript (probeset: 225210_S_AT) from a panel of 59 breast cancer patients whose transcript profile was previously analyzed on a breast carcinoma microarray $(29,30)$. As shown in Fig. 4C, the RPS6KA2 transcript and its antisense RNA proved to be negatively correlated with a high confidence $(\mathrm{p}=0.0092)$ in 42 out of 59 patients $(71 \%)$. Taken together, this evidence further supports the hypothesis of functional regulation of sense/antisense transcript for RPS6KA2 in breast cancer.

Significantly, RPS6KA2 was recently reported as a tumor suppressor gene for sporadic epithelial ovarian carcinoma, thus strengthening its functional relevance for the pathogenesis of several cancer types (8). Monoallelic expression for this gene was also reported by the same authors, providing an indirect support for the occurrence of antisense RNA-mediated regulation of this gene, since monoallelic expression of several imprinted genes within mammalian genomes has been functionally linked to the presence of antisense transcription (37).

A further characterization of the sense-antisense transcript interaction in a wide range of normal and neoplastic tissues is therefore warranted for this gene, in order to better define both the molecular mechanisms by which its expression is downregulated in cancer cells and its involvement in a wider range of cancer types besides ovarian and breast carcinomas.

\section{Discussion}

The recent completion of the human genome sequence provided the scientific community with new tools to better understand the mechanisms of gene regulation at the genomic level. In this research area, the growing interest that has been recently devoted to the study of natural antisense transcript (NATs) stems based on two arguments: first, recent in silico and experimental evidence suggests that a significant fraction of the mammalian transcripts represents NATs (18-20). Second, there is increasing evidence that NATs can provide the cell with a versatile tool for carrying out gene regulation by means of several mechanisms. Cancer is, among other diseases, one of the most important pathological conditions which have been proven to be highly sensitive to abnormal gene expression patterns.

Indeed, several molecular mechanisms of aberrant gene expression such as gene amplification, epigenetic silencing and altered gene dosage due to aneuploidy have been defined as important contributors to tumor progression $(21,38,39)$. Moreover, experimental and bioinformatic evidence has been recently reported to suggest the involvement of antisense transcription in the pathogenesis of cancer. For instance, by using prostate cancer as a model, Reis et al reported an increased expression of intronic transcripts correlating to the degree of tumor differentiation when compared to non-intronic regions. Strikingly, a significant fraction of these intronic transcripts was represented by antisense RNAs (40). As a further example, the longer than average length of 5'-UTR and first exon which has been recently reported in genes which have overlapping antisense transcripts in the 5 ' region and are known to be silenced in human cancer has been taken as indirect evidence that antisense transcription could play a pivotal role in such epigenetic phenomenon (41).

Finally, a recent study by $\mathrm{Yu}$ et al provided striking evidence for the occurrence of antisense RNA-mediated 
regulation as a mechanism to control the expression of known tumor suppressor genes (24).

Altogether, the above-mentioned data suggest that antisense-mediated regulation of gene expression has been largely under-estimated in the past as a putative molecular mechanism involved in cancer pathogenesis or progression. Indeed, as far as the identification of tumor suppressor genes is concerned, the long established strategy of using $\mathrm{LOH}$ data combined with mutation screening to map and identify these cancer-related genes has proven unsuccessful in many instances (42), thus suggesting that novel conceptual avenues are needed to identify most of the still elusive oncosuppressor genes scattered throughout the human genome. Significantly, antisense-mediated gene silencing has been recently shown to provide a powerful tool for the identification of tumor suppressor genes. For instance, Li et al were able to demonstrate the oncosuppressive role for the utrophin gene by a functional assay based on antisense-mediated suppression of the endogenous sense gene transcript from this locus in a cancer cell line (43). Despite this suggestive evidence, to date little effort has been devoted to a better understanding of the role that antisense-mediated regulation of gene expression play in the pathogenesis of cancer.

In this work, we predicted in silico a significant number of putative NATs for several annotated genes mapping to two chromosome 6 genomic regions whose alterations are associated with a wide range of human malignancies. We were able to experimentally confirm antisense transcription for $69 \%$ of the predicted sense-antisense gene pairs by means of strand-specific RT-PCR with just a single tissue (human fetal brain) as a source of template RNA.

Within our panel of experimentally validated sense/antisense gene pairs, the exon overlapping (AS) and non-exon overlapping (NOBs) categories were represented with similar frequencies. This was not an unexpected result, since an increasing number of recent whole-genome studies have reported the widespread occurrence of intronic antisense transcription in the human genome (44). Co-expression of both members of a sense-antisense gene pair was observed with a high frequency in our panel of candidate genes, suggesting that some of these gene pairs could be engaged in antisense-mediated cross-regulation in vivo.

Regarding this issue, Chen et al have recently proposed three main criteria to define sense-antisense gene pairs for which antisense-mediated control of gene expression is likely to occur (33). Two of these criteria (i.e. evolutionary conservation of a sense-antisense gene pair and coexpression of sense-antisense transcripts pair within the same cell type) were shown to apply to several sense-antisense gene pairs identified in this work, supporting the notion that some of these NATs could indeed play a role in the regulation of the expression levels of the sense partner. Interestingly, both criteria were also fulfilled for 5 genes potentially involved in cancer pathogenesis (or whose annotations predict a role in tumorigenesis) (Table II).

Regarding the third criterion defined by Chen et al (inverse expression levels for the sense and antisense transcripts within a particular locus), our quantitative real-time PCR assays strongly suggested that such a phenomenon actually occurs for one of the five putative cancer-related genes (RPS6KA2) that were investigated in detail, thus defining this gene as a strong candidate for antisense RNAmediated gene regulation. Indeed, RNAi assays coupled to standard gene overexpression studies provided clear evidence for the occurrence of regulatory crosstalk between RPS6KA2 sense and its antisense transcript into a single breast cancer line. Whether such regulatory mechanisms occur in other breast cancer cell lines is currently unknown, and our future work will be focused to this issue.

It is noteworthy that RPS6KA2 was recently described as a tumor suppressor gene for sporadic ovarian carcinoma (8), thus providing further support for the relevance of this gene in cancer pathogenesis. Indeed, our preliminary results on a panel of human ovarian cancer cell lines suggest that antisensemediated regulation of this gene likely takes place also in the setting of this cancer type (LM and FA, unpublished data). The fact that RPS6KA2 was recently reported as a tumor suppressor involved in a cancer type (ovarian carcinoma) different from the one investigated here strongly supports the usefulness of antisense transcription mapping in cancer-related genomic regions as a tool for the identification of genes whose changes in expression levels are somehow related to cancer development or progression. At the same time, the antisense-mediated regulation of the RPS6KA2 reported in this work could contribute to shed more light on the molecular mechanisms involved in the functional inactivation of this gene in human cancer.

Our data allowed us to draw a striking parallel with those recently reported by $\mathrm{Yu}$ et al (24) in several respects. First, an inverse expression between the transcript levels of a TSG and its NAT was observed in our study on both cancer cell lines and primary tumor samples. Second, forced antisense overexpression produced a marked decrease in the corresponding sense transcript in vivo. Thus, RPS6KA2 can be defined as a bona fide member of the growing family of TSG whose cancer-associated expression changes are mediated by antisense transcription.

As far as the molecular mechanism by which antisensemediated regulation of RPS6KA2 gene expression takes place in vivo, it is worth noting that this sense/antisense gene pair does not show any exon overlap. Therefore, if the underlying regulatory mechanism requires dsRNA formation (as the results of our antisense overexpression assay suggests) then an interaction between RPS6KA2 pre-mRNA and its antisense transcript has to be postulated. However, other known dsRNA-independent regulatory pathways, such as epigenetic anti-sense-mediated silencing or transcriptional collision between two converging RNA polymerase II complexes (15) might also be invoked to explain the observed inverse expression pattern within this gene pair. Such issue is currently being investigated in our laboratory.

In conclusion, the combination of in silico analysis and primary validation of antisense transcripts allowed us to identify a subset of genes that display antisense transcription and are annotated with molecular or cellular functions compatible with a role in cancer pathogenesis. Among these genes, RPS6KA2 stood out as a plausible antisense-regulated TSG. To our knowledge, this is one of the first attempts to get a comprehensive picture of sense-antisense transcription from a particular cancer-related genomic region. We believe 
that a more widespread application of this strategy will provide an important contribution to the long-term goal of producing a molecular blueprint of human cancer.

\section{Acknowledgements}

The sequence-related part of this work was conducted on an HP rx4640 Itanium2 server granted to IFOM in the framework of the 'HP Integrity' program coordinated by A.G. M.C. is a participant of the Doctorate in Molecular Medicine at the University of Milan. Research in the lab of G.L. was supported by a grant of Fondazione Cariplo (2004/1530) and by a grant from MIUR (RBLA039M7M). R.T. and F.A. were supported by the Academic Research Fund (FAR) from the Insubria University.

\section{References}

1. Zhang Y, Matthiesen P, Harder S, et al: A 3-cM commonly deleted region in $6 \mathrm{q} 21$ in leukemias and lymphomas delineated by fluorescence in situ hybridization. Genes Chromosomes Cancer 27: 52-58, 2000

2. Tibiletti MG, Trubia M, Ponti E, et al: Physical map of the D6S149-D6S193 region on chromosome 6q27 and its involvement in benign surface epithelial ovarian tumours. Oncogene 16: 1639-1642, 1998.

3. Miele ME, Jewett MD, Goldberg SF, et al: A human melanoma metastasis-suppressor locus maps to $6 \mathrm{q} 16.3-\mathrm{q} 23$. Int J Cancer 86: 524-528, 2000

4. Trent JM, Stanbridge EJ, McBride HL, et al: Tumorigenicity in human melanoma cell lines controlled by introduction of human chromosome 6. Science 247: 568-571, 1990.

5. Negrini M, Sabbioni S, Possati L, Rattan S, Corallini A, Barbanti-Brodano G and Croce CM: Suppression of tumorigenicity of breast cancer cells by microcell-mediated chromosome transfer: studies on chromosomes 6 and 11. Cancer Res 54: 1331-1336, 1994.

6. Theile M, Seitz S, Arnold W, et al: A defined chromosome 6q fragment (at D6S310) harbors a putative tumor suppressor gene for breast cancer. Oncogene 13: 677-685, 1996.

7. Pasqualucci L, Compagno M, Houldsworth J, et al: Inactivation of the PRDM1/BLIMP1 gene in diffuse large B cell lymphoma. J Exp Med 203: 311-317, 2006.

8. Bignone PA, Lee KY, Liu Y, et al: RPS6KA2, a putative tumour suppressor gene at $6 \mathrm{q} 27$ in sporadic epithelial ovarian cancer. Oncogene 26: 683-700, 2007.

9. Acquati F, Morelli C, Cinquetti R, et al: Cloning and characterization of a senescence inducing and class II tumor suppressor gene in ovarian carcinoma at chromosome region 6q27. Oncogene 20: 980-988, 2001.

10. Steinemann D, Gesk S, Zhang Y, et al: Identification of candidate tumor suppressor genes in 6 q27 by combined deletion mapping and electronic expression profiling in lymphoid neoplasms. Genes Chromosomes Cancer 37: 421-426, 2003.

11. Sinclair PB, Sorour A, Martineau M, et al: A fluorescence in situ hybridization map of $6 \mathrm{q}$ deletions in acute lymphocytic leukemia: identification and analysis of a candidate tumor suppressor gene. Cancer Res 64: 4089-4098, 2004.

12. Rutherford S, Yu Y, Rumpel CA, Frierson HF Jr and Moskaluk CA: Chromosome 6 deletion and candidate tumor suppressor genes in adenoid cystic carcinoma. Cancer Lett 236 : 309-317, 2006

13. Zhang L, Anglesio MS, O'Sullivan M, et al: The E3 ligase HACE1 is a critical chromosome 6q21 tumos suppressor involved in multiple cancers. Nat Med 13: 1060-1069, 2007.

14. Lavorgna G, Dahary D, Lehner B, Sorek R, Sanderson CM and Casari G: In search of antisense. Trends Biochem Sci 2: 88-94, 2004.

15. Lapidot $M$ and Pilpel $Y$ : Genome-wide natural antisense transcription: coupling its regulation to its different regulatory mechanisms. EMBO Rep 7: 1216-1222, 2006.

16. Shendure J and Church GM: Computational discovery of senseantisense transcription in the human and mouse genomes. Genome Biol 3: 22, 2002.
17. Yelin R, Dahary D, Sorek R, et al: Widespread occurrence of antisense transcription in the human genome. Nat Biotechnol 21: 379-386, 2003.

18. Chen J, Sun M, Kent WJ, et al: Over $20 \%$ of human transcripts might form sense-antisense pairs. Nucleic Acids Res 32: 4812-4820, 2004.

19. Engstrom PG, Suzuki H, Ninomiya N, et al: Complex loci in human and mouse genomes. PLoS Genet 2, e47: 564-577, 2006.

20. Katayama S, Tomaru Y, Kasukawa T, et al: Antisense transcription in the mammalian transcriptome. Science 309: 1564-1566, 2005.

21. Schwab M: Amplification of oncogenes in human cancer cells Bioessays 20: 473-479, 1998 .

22. Laird PW: Cancer epigenetics. Hum Mol Genet 14: R65-R76, 2005.

23. Tufarelli C, Stanley JA, Garrick D, Sharpe JA, Ayyub H, Wood WG and Higgs DR: Transcription of antisense RNA leading to gene silencing and methylation as a novel cause of human genetic disease. Nat Genet 34: 157-165, 2003.

24. Yu W, Gius D, Onyango P, et al: Epigenetic silencing of tumour suppressor gene p15 by its antisense RNA. Nature 451: 202-206, 2007

25. Moorwood K, Charles AK, Salpekar A, Wallace JI, Brown KW and Malik K: Antisense WT1 transcription parallels sense mRNA and protein expression in fetal kidney and can elevate protein levels in vitro. J Pathol 185: 352-359, 1998.

26. Rossignol F, Vache $\mathrm{C}$ and Clottes E: Natural antisense transcripts of hypoxia-inducible factor 1alpha are detected in different normal and tumour human tissues. Gene 299: 135-140, 2002.

27. Corcoran MM, Hammarsund M, Zhu C, et al: DLEU2 encodes an antisense RNA for the putative bicistronic RFP2/LEU5 gene in humans and mouse. Genes Chromosomes Cancer 40: 285-297, 2004.

28. Lavorgna G, Sessa L, Guffanti A, Lassandro L and Casari G: AntiHunter: searching BLAST output for EST antisense transcripts. Bioinformatics 20: 583-585, 2004.

29. Vecchi M, Confalonieri S, Nuciforo P, et al: Breast cancer metastases are molecularly distinct from their primary tumors. Oncogene 27: 2148-2158, 2008 .

30. Nicassio F, Bianchi F, Capra M, et al: A cancer-specific transcriptional signature in human neoplasia. J Clin Invest 115 : 3015-3025, 2005

31. Galante PA, Vidal DO, De Souza JE, Camargo AA and De Souza SJ: Sense-antisense pairs in mammals: functional and evolutionary considerations. Genome Biol 8: R40, 2007.

32. Thierry-Mieg D and Thierry-Mieg J: AceView: a comprehensive cDNA-supported gene and transcripts annotation. Genome Biol 7 (Suppl. 1): S12, 2006.

33. Chen J, Sun M, Hurst LD, Carmichael GG and Rowley JD: Genome-wide analysis of coordinate expression and evolution of human cis-encoded sense-antisense transcripts. Trends Genet 21: 326-329, 2005

34. Dahary D, Elroy-Stein O and Sorek R: Naturally occurring antisense: transcriptional leakage or real overlap? Genome Res 15: 364-368, 2005.

35. Boguski MS and Jones AR: Neurogenomics: at the intersection of neurobiology and genome sciences. Nat Neurosci 7: 429-433, 2004.

36. Kiyosawa H, Mise N, Iwase S, Hayashizaki Y and Abe K: Disclosing hidden transcripts: mouse natural sense-antisense transcripts tend to be poly(A) negative and nuclear localized. Genome Res 4: 463-474, 2005.

37. Rougeulle $\mathrm{C}$ and Heard E: Antisense RNA in imprinting: spreading silence through Air. Trends Genet 18: 434-437, 2002.

38. Laird PW: Cancer epigenetics. Hum Mol Genet 14: R65-R76, 2005.

39. Duesberg P, Li R, Fabarius A and Hehlmann R: The chromosomal basis of cancer. Cell Oncol 27: 293-318, 2005.

40. Reis EM, Nakaya HI, Louro R, et al: Antisense intronic noncoding RNA levels correlate to the degree of tumor differentiation in prostate cancer. Oncogene 23: 6684-6692, 2004.

41. Finocchiaro G, Carro MS, Francois S, Parise P, Di Ninni V and Muller H: Localizing hotspots of antisense transcription. Nucleic Acids Res 35: 1488-1500, 2007.

42. Tomlinson IP, Lambros MBK and Roylance RR: Loss of heterozygosity analysis: practically and conceptually flawed? Genes Chromosomes Cancer 34: 349-353, 2002.

43. Li Y, Huang YL, He J, Wang W, Davies KE, Nosè V and Xiao S: UTRN on chromosome $6 \mathrm{q} 24$ is mutated in multiple tumors. Oncogene 26: 6220-6228, 2007.

44. Reis EM, Louro R, Nakaya HI and Verjovski-Almeida S: As antisense RNA gets intronic. OMICS 9: 2-12, 2005. 